Cite this: Phys. Chem. Chem. Phys., 2013,

\title{
Eco-solvents - cluster-formation, surfactantless microemulsions and facilitated hydrotropy
}

15, 10971

Received 12th February 2013, Accepted 29th April 2013

DOI: $10.1039 / \mathrm{c} 3 \mathrm{cp} 50636 \mathrm{c}$

www.rsc.org/pccp

\author{
Michael L. Klossek, Didier Touraud and Werner Kunz*
}

\begin{abstract}
In this paper we consider clusters in the ternary systems water-benzyl alcohol and ethanol, ethyl lactate or $\gamma$-valerolactone as found with the help of dynamic and static light scattering experiments. These ternary mixtures are powerful solvent media and consist only of low-toxic solvents of natural origin. In a recent work we have shown that surfactantless microemulsions are formed in the water-ethanol- $n$ octanol system. By contrast, in the systems studied here the sizes of the aggregates are too small to be considered as micelles. It can be postulated that the presence of clusters or larger structures as in surfactantless microemulsions is strongly influenced by the most hydrophobic compound. The phenomenon of facilitated hydrotropy is also investigated in the above-mentioned systems. In this context, ethanol is considered as the primary hydrotrope and the more hydrophobic benzyl alcohol as the facilitating secondary hydrotrope. The hydrophobic dye Disperse Red 13 is used as a marker of facilitated hydrotropy. The results suggest that the degree of self-association of eco-solvent has a significant influence on the hydrotropic efficiency of benzyl alcohol.
\end{abstract}

\section{Introduction}

Principle 2 of the six principles of green extractions, as defined by Chemat, ${ }^{1}$ refers to the use of principally water or agro-solvents stemming from agricultural resources. Bio- or agro-based solvents (eco-solvents) are important classes of solvents that may replace solvents obtained from petrochemistry at least for some applications. Eco-solvents can be gained from biomass, such as wood, starch, vegetable oils and fruits. Nature produces 170 billion tons of biomass per year by photosynthesis. $75 \%$ belongs to the class of carbohydrates, and just $3-4 \%$ of these compounds are used by humans for food and nonfood purposes. ${ }^{2}$ Ethanol, ethyl lactate and $\gamma$-valerolactone are three examples of eco-solvents derived from carbohydrates (sugars). Capello et al. ${ }^{3}$ tried to give a definition of green solvents. One of the four main points which directs to green solvents was to use bio- or eco-solvents derived from renewable feedstock. They explicitly mention the production of ethanol by fermentation. The other three points were the substitution of hazardous solvents, the use of supercritical fluids or ionic liquids. In another work, Moity et al. used the COnductorlike Screening MOdel for Real Solvents (COSMO-RS) to classify sustainable solvents. ${ }^{4}$ It should help to find green alternatives for organic solvents.

Institute of Physical and Theoretical Chemistry, University of Regensburg, 93040 Regensburg, Germany. E-mail: werner.kunz@chemie.uni-regensburg.de; Fax: +49 941943 4532; Tel: +499419434044
Ethanol (EtOH) is the most common eco-solvent. The world production of bioethanol is estimated to be 22000 millions of US liquid gallons per year. ${ }^{5}$ It can be produced by fermentation of sugar-rich materials such as sugar crops (sugar cane and sugar beet), starch crops (corn), or cellulosic feedstocks (wood, grasses and agricultural residues). ${ }^{6,7}$ Although EtOH is flammable and produces explosive emissions, it is used on a large scale because of its availability in high purity, its low price and low viscosity. Of course, it is completely biodegradable. Its boiling point is $78{ }^{\circ} \mathrm{C}$.

Ethyl lactate (EL) is an environmentally acceptable solvent. Moreover, it is biodegradable and has a low viscosity. It is not a volatile organic compound (VOC). A way of producing EL is the esterification of lactic acid with EtOH. ${ }^{8}$ The advantage of this process is that both reactants are obtained from renewable resources. The selling price for commonly used solvents in the USA is around $\$ 0.90$ to $\$ 1.70$ per pound. Processing advances have lowered the price of EL from $\$ 1.50$ to $\$ 2.00$ per pound to $\$ 0.85$ to $\$ 1.00$ per pound what makes it more competitive to petro-solvents. ${ }^{7,9}$ Its low melting $\left(-26{ }^{\circ} \mathrm{C}\right)$ and high boiling points $\left(151-155^{\circ} \mathrm{C}\right)$ are also advantageous.

$\gamma$-Valerolactone (GVL) is another sugar-based eco-solvent that has attracted attention recently. ${ }^{10-14}$ It can be produced at yields higher than $95 \%$ by the hydrogenation of levulinic acid ${ }^{15,16}$ which can be derived from biomass. ${ }^{17,18}$ Horváth et al. showed recently that GVL is a promising green and sustainable solvent with interesting properties such as low melting $\left(-31^{\circ} \mathrm{C}\right)$ 


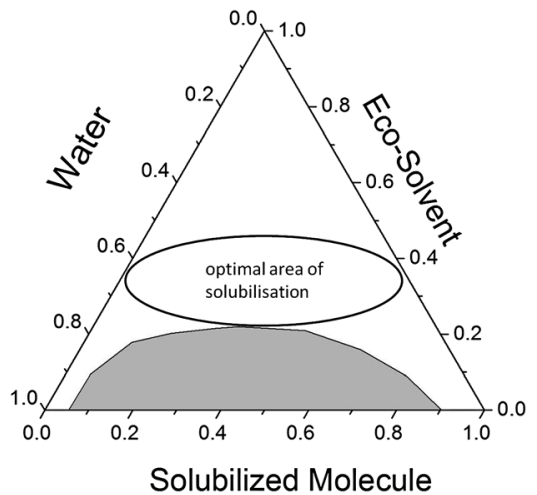

Fig. 1 Illustration of the optimal region in the ternary phase diagram to solubilize a hydrophobic molecule with the minimum of co-solvent. This region is also characterised by optimum structuring. The white zone represents monophasic systems, the grey zone biphasic ones. The compositions are given in mass ratios.

and high boiling $\left(207^{\circ} \mathrm{C}\right)$ points, a very low vapour pressure $\left(0.65 \mathrm{kPa}\right.$ at $\left.25{ }^{\circ} \mathrm{C}\right)$, no formation of measurable amount of peroxides, etc. ${ }^{11}$

In a recent work we showed that environmentally friendly microemulsions can be made without surfactants. ${ }^{19}$ The condition for this phenomenon, the so-called "pre-ouzo" effect, is that at least three compounds are mixed where solvent $\mathrm{A}$ is miscible with solvent $\mathrm{B}$, solvent $\mathrm{B}$ is miscible with compound $\mathrm{C}$, and $\mathrm{A}$ and $\mathrm{C}$ are immiscible. Surfactantless microemulsions have already been known since the late 1970s but to date, only few articles deal with this topic and none of them deliver a satisfactory explanation. ${ }^{20-23}$ In our previous work we showed that most pronounced structuring in terms of microemulsions is found close to the demixing (binodal) line, see Fig. 1. This is also the region where industrial extraction processes are made, and it is supposed that structuring is most important for efficient extraction. ${ }^{24}$ Durand et al. ${ }^{25}$ investigated the system water-benzyl alcohol (BA)-dimethyl isosorbide (DMI), the latter being also an eco-solvent derived from sugar, using dynamic light scattering (DLS). Their results concerning the occurrence of significant correlation functions are comparable to the results that we found in our studies mentioned before. Concerning their model system they were not able to find a reasonable explanation for the formation of aggregates. Instead they focused on the concept of facilitated hydrotropy, which may have its origin in aggregate formation. The concept of facilitated hydrotropy consists of solubilizing an insoluble amphiphilic compound in water in the presence of a co-solvent, which acts like a hydrotrope, and a further more hydrophobic compound, the facilitating hydrotrope. ${ }^{26,27}$ A hydrotrope solubilises hydrophobic compounds in aqueous solutions. Usually it has a hydrophilic and a hydrophobic part, like a surfactant, but no spontaneous self-aggregation occurs. A facilitating hydrotrope is often a partially water-soluble aromatic molecule (such as BA, benzoate, or benzoic acid) which can be completely solubilised in water with a water-soluble co-solvent (such as EtOH). When the aromatic compound is solubilised, it transforms from a partially water-soluble solute to a solubilising agent. In the present study we investigate the systems
water-BA-eco-solvent (EtOH, EL, GVL) with static (SLS) and dynamic (DLS) light scattering to determine the structure. Further, we look for the possibility of facilitated hydrotropy. BA was chosen as a model of a hydrophobic hydrotrope molecule to be solubilised in the binary water-eco-solvent mixture. In order to study facilitated hydrotropy Disperse Red 13 (DR-13) was used as a hydrophobic molecule (octanol-water distribution $\log p=5.2^{28}$ ). According to the concept of facilitated hydrotropy, ${ }^{29} \mathrm{BA}$ is the facilitating hydrotrope and the eco-solvents are hydrotropes to solubilise BA in the aqueous system. The work is part of a research program, initiated in our institute, aiming at a better understanding of solubilisation media and their mesoscopic structuring to optimise solvents and extraction media.

\section{Experimental}

\subsection{Chemicals}

Ethyl lactate (purity $=98 \%$ ), $\gamma$-valerolactone $(99 \%)$, and Disperse Red 13 (DR-13, dye content 95\%, structure is shown in Fig. 2) were purchased from Sigma-Aldrich (Steinheim, Germany). Ethanol (99.9\%) was purchased from Baker (Deventer, Netherlands) and benzyl alcohol ( $\geq 99 \%$ ) from Merck (Hohenbrunn, Germany). All chemicals were used without further purification. All solutions were prepared using water with a resistivity of $18 \mathrm{M} \Omega \mathrm{cm}$.

\subsection{Methods and techniques}

2.2.1 Phase diagrams. The phase diagrams were recorded using a dynamic and static process according to Clausse et al. ${ }^{30}$ In screwable tubes the binary mixtures at different concentrations were thermostated at $25{ }^{\circ} \mathrm{C}$ in a thermostatically controlled test tube rack. Then, the third component was added successively using Eppendorf pipettes until a change in the phase behavior occurred. The phase transition was determined with the naked eye. When a change in the phase behavior was observed, the weight fractions of the added component were derived from precise weight measurements. The composition of the solution was then calculated from the masses of all components. It was estimated that the accuracy of the compositions for the transparency-to-turbidity transition was better than $2 \%$.

2.2.2 UV/Vis measurements. All experiments concerning the solubilisation process were done in a thermostated room at $25{ }^{\circ} \mathrm{C}\left( \pm 0.2^{\circ} \mathrm{C}\right)$. The solutions containing water and the studied components were prepared at appropriate concentration. Then, DR-13 was added until saturation was reached, i.e. some dye remained undissolved, and left equilibrated for $24 \mathrm{~h}$.

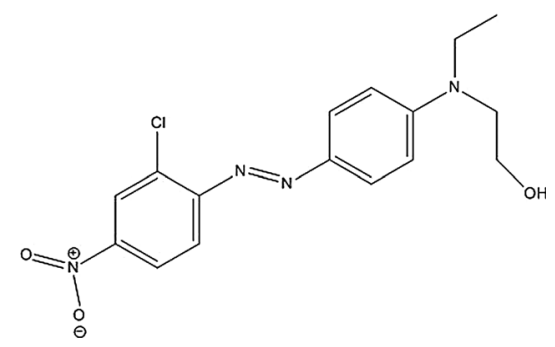

Fig. 2 Chemical structure of DR-13. 
The solutions were then filtered using a syringe equipped with a $0.45 \mu \mathrm{m}$ PTFE membrane filter in order to separate the nonsolubilised excess of dye from the solutions. The optical densities (O.D.) of the filtered solutions were measured in $1 \mathrm{~cm}$ path length cells using a UV-visible spectrophotometer Cary-3E. DR-13 has an absorption maximum at a wavelength of $525 \mathrm{~nm}$. When the absorbance was higher than one, the sample was diluted with the same solution without dye.

\subsubsection{Light scattering}

Dynamic light scattering. Dynamic light scattering (DLS) experiments were performed using a goniometer CGS-II from ALV (Langen, Germany). The goniometer was equipped with an ALV-7004/Fast Multiple Tau digital correlator and a verticalpolarised $22 \mathrm{~mW}$ HeNe-laser (wavelength $\lambda=632.8 \mathrm{~nm}$ ). All measurements were done at a scattering angle of $90^{\circ}$ after thermostating to $25^{\circ} \mathrm{C}$. Before the measurements, all solutions were filtered using a $0.2 \mu \mathrm{m}$ PTFE membrane filter to remove all dust particles. Then, the samples were transferred to a cylindrical light-scattering cell of $10 \mathrm{~mm}$ outer diameter. The measurement time was $300 \mathrm{~s}$. The obtained correlation functions were fitted with the software Table Curve 2D v5.01 by a monomodal equation. This function can be described as follows:

$$
y=a_{0}+\left(a_{1} \mathrm{e}^{-a_{2} X}\right)^{2}
$$

$a_{0}$ is a constant base value, usually $1, a_{1}$ refers to the dynamic part of the amplitude and $a_{2}$ is the decay rate linked to the diffusion coefficient $D$ :

$$
a_{2}=D q^{2}
$$

$q$ is the scattering vector which is defined as

$$
q=\frac{4 \pi n}{\lambda} \sin \frac{\theta}{2}
$$

In this equation, $n$ is the refractive index, $\lambda$ the wavelength of the irradiated light, and $\theta$ the detection angle. The hydrodynamic radius $R_{\mathrm{h}}$ can be derived from the Stokes-Einstein equation:

$$
D=\frac{k_{\mathrm{b}} T}{6 \pi \eta R_{\mathrm{h}}}
$$

For the precise calculation of the hydrodynamic radius $R_{\mathrm{h}}$ the knowledge of the dynamic viscosities, $\eta$ of the solutions is indispensable (see eqn (4)). The viscosities were measured using the automated rolling ball viscometer AMVn from Anton Paar (Graz, Austria) at $25{ }^{\circ} \mathrm{C}$ using a calibrated glass capillary with $1.6 \mathrm{~mm}$ diameter and corresponding steel balls. The relative uncertainty of $\eta$ is approx. $0.5 \%$. As shown in eqn (3), the refractive index $n$ is another important variable. This was determined using an Abbemat WR from Anton Paar (Graz, Austria) at $25{ }^{\circ} \mathrm{C}$ and a wavelength $\lambda$ of $589 \mathrm{~nm}$.

Static light scattering. Static light scattering experiments were performed using the same apparatus as described above. Measurements were conducted at $25{ }^{\circ} \mathrm{C}$ and at scattering angles of $45^{\circ}, 90^{\circ}$ and $135^{\circ}$. Before the measurements the samples were treated in the same way as for DLS experiments.
The apparent molar mass obtained from SLS via the Rayleigh relation:

$$
\frac{\Re}{K C}=M S(q)=M_{\mathrm{app}},
$$

where $\Re$ is the Rayleigh ratio in $\mathrm{m}^{-1}$ (Rayleigh ratio standard taken from ref. 31 and 32), $S(q)$ is the structure factor which expresses the influence of micelle-micelle (or more general object-object) interactions on the scattering, $C$ is the concentration in $\mathrm{g} \mathrm{L}^{-1}, M$ the molar mass of the micelles and $M_{\text {app }}$ the corresponding apparent mass. $K$ is defined as

$$
K=4 \pi^{2} n^{2}\left(\frac{\mathrm{d} n}{\mathrm{~d} c}\right)^{2} \lambda^{-4} N_{\mathrm{A}}^{-1}
$$

In this equation $n$ is again the refractive index, $\frac{\mathrm{d} n}{\mathrm{~d} c}$ the refractive index increment in $\mathrm{L} \mathrm{g}^{-1}$, which was obtained by precise refractive index measurements as described above, $\lambda$ the wavelength in $\mathrm{m}$ and $N_{\mathrm{A}}$ is the Avogadro number. The Rayleigh ratio of particles in solution can be calculated using the following equation:

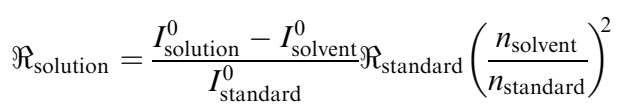

with $I^{0}$ being the scattered intensity of the solvent, solution or the used standard (in our case toluene). $\left(\frac{n_{\text {solvent }}}{n_{\text {standard }}}\right)^{2}$ is the correction factor of the scattered volume, which is often introduced due to different diffraction effects between the sample and the standard. ${ }^{33}$ In first approximation we set this term equal to one. The spherical volume $\left(V_{\text {sphere }}\right)$ can be obtained from $M_{\text {app }}$ according to eqn (8).

$$
V_{\text {sphere }}=\frac{M_{\text {app }}}{N_{\mathrm{A}} \rho_{\text {particle }}}=\frac{4}{3} \pi r^{3}
$$

The problem was to find an expression for $\rho_{\text {particle. Due to }}$ the similarity of the densities of the involved fluids, the density of the eco-solvent for the corresponding system was used.

\section{Results and discussion}

\subsection{Ternary phase diagrams}

For a better understanding of facilitated hydrotropy the phase behaviour of the ternary systems water-BA-eco-solvent was studied first. In Fig. 3 the three ternary phase diagrams are shown. Two distinct areas can be found, a clear transparent single phase and a turbid two-phase zone. All three eco-solvents are completely soluble in water and BA. Moreover, the three eco-solvents increase the cosolubility of water and BA significantly. Above a certain mass fraction of co-solvent $(0.25$ for $\mathrm{EtOH}, 0.35$ for EL, and 0.55 for GVL), only a monophasic system is obtained for every water/BA ratio.

\subsection{Light scattering experiments}

Fig. 4 presents selected self-correlation functions from DLS along lines with increasing BA content for the systems water-BA-EtOH, 


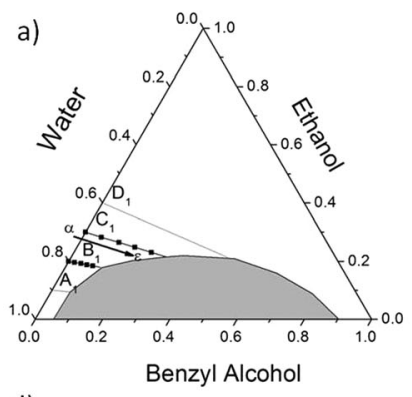

d)

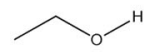

Ethanol

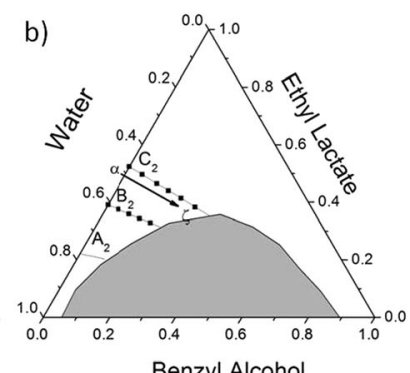

e)

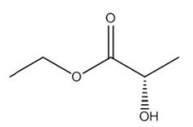

Ethyl Lactate

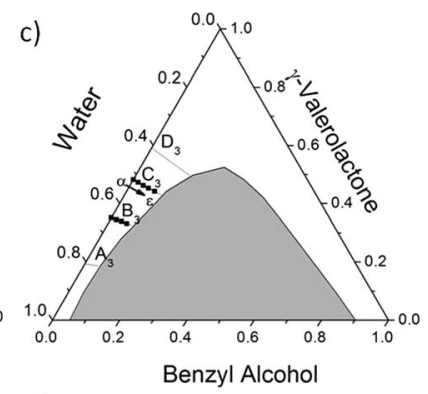

f)

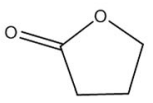

$\gamma$-Valerolactone

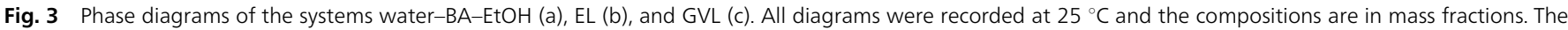

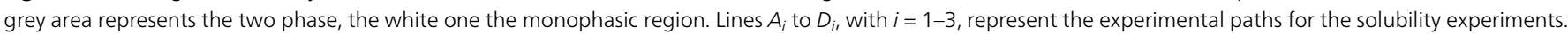

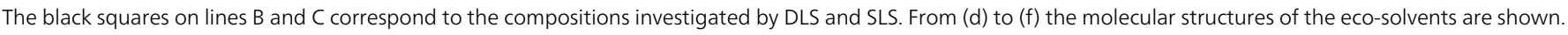
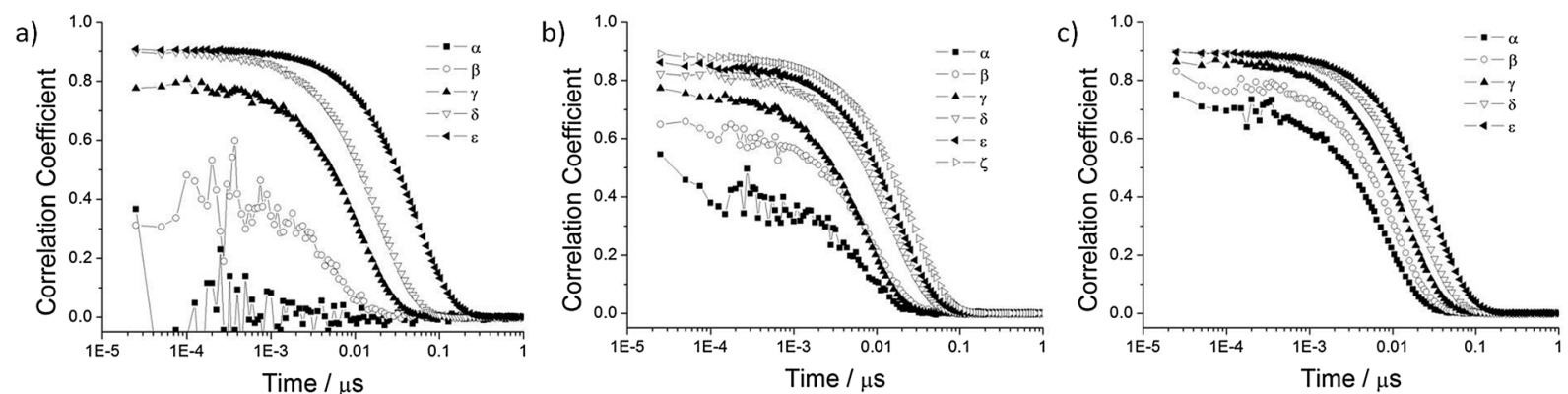

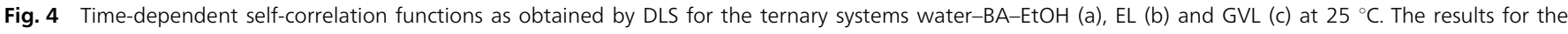
different compositions $\alpha-\varepsilon, \zeta$ on the experimental paths $C_{i}$ of Fig. 1 are presented.

EL, and GVL. Two phenomena can be distinguished. For EtOH and EL, the correlation becomes more and more pronounced, the closer the composition is to the phase separation composition. On the other hand, the correlation function of the binary system water-GVL is already significant. With the assumption of a spherical symmetry, the deduced radii of the particles get larger the closer the composition is to the phase boundary. Even relatively far away structures clearly occur, a fact that let us assume that these correlations are not a critical phenomenon with large fluctuations close to a critical point. Comparing the three curves $\alpha$ from Fig. 4(a)-(c) which correspond to the binary system water-eco-solvent it can be seen that $\mathrm{EtOH}$ in water does not produce a significant correlation function. In the case of EL the formation of aggregates can be assumed, but only the correlation functions of the binary water-GVL can be properly fitted.

But DLS results alone are not a definitive argument for spherical structures in solution. Therefore, SLS measurements were performed at three angles $\left(45^{\circ}, 90^{\circ}\right.$ and $\left.135^{\circ}\right)$ to rule out the possibility of critical fluctuations (Ornstein-Zernicke scattering) which would give a strong angle dependence. The radii as derived from Rayleigh scattering at the three angles mentioned before did not differ by more than $1 \%$. In Fig. 5 (b) to (d) the radii derived from DLS and SLS with the assumption of spherical particles are shown as a function of the BA mass fraction. In addition, Fig. 5(a) shows the graph obtained from the system water-ethanol- $n$-octanol from a previous work. ${ }^{19}$ In contrast to the three curves mentioned before this figure is an example of a system with well-defined micelles. The difference to our system is the overall hydrodynamic radii measured. For the ecosolvents the objects are very small (almost always below $1 \mathrm{~nm}$ ). In this case we cannot speak of well-defined micelles in the systems, but of cluster formation of the solvent molecules or of a pre-micellar area in the phase diagram. Though the particle sizes are very low, all three curves show a satisfactory agreement between the radii obtained from both techniques. This is a strong hint at the presence of more or less spherical clusters. This procedure of comparing DLS and SLS results has already identified micelles of defined sizes ${ }^{34}$ and pre-micelles with strongly concentration dependent aggregation numbers for short chain surfactants. ${ }^{35}$

\subsection{Solubilisation experiments}

To study the concept of facilitated hydrotropy, the solubilisation of a hydrophobic dye, DR-13, as a function of the BA concentration was investigated. For this purpose, the water-to-eco-solvent mass ratio was kept constant and the amount of BA was successively increased. The compositions used for these experiments are 
a)
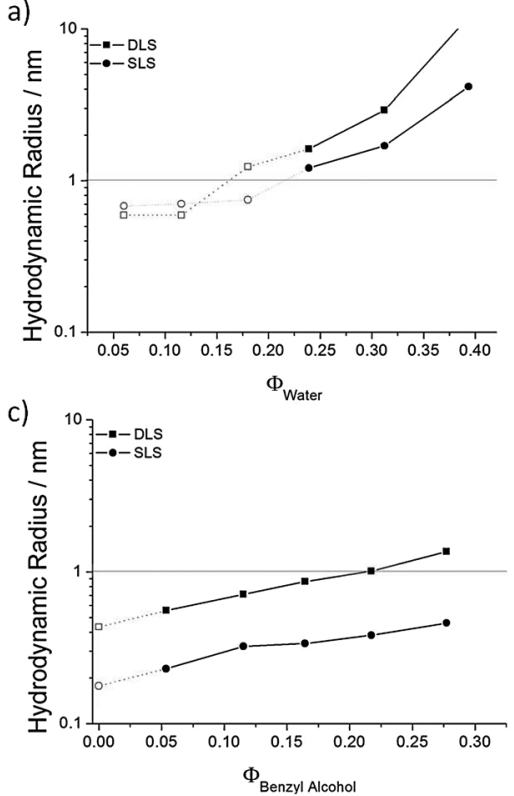

b)
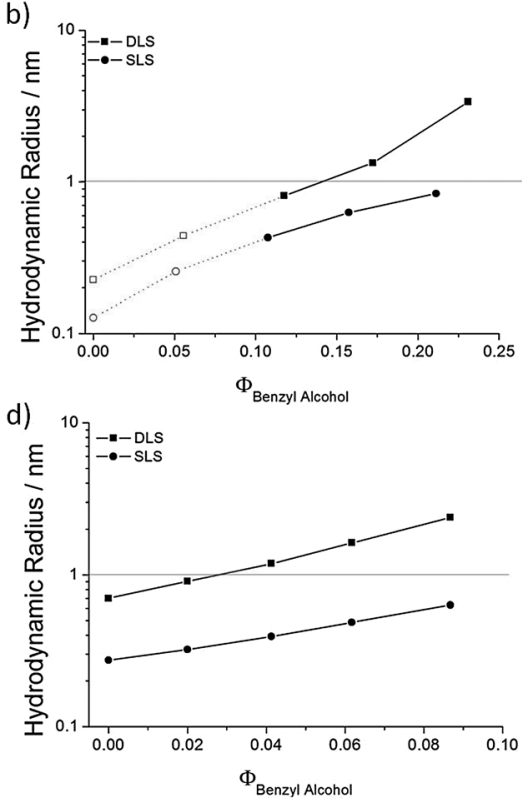

Fig. 5 Estimated radii of scattering objects inferred from DLS (black squares) and SLS (black circles) assuming spherical geometry for the systems (a) water-ethanol-noctanol and water-BA-EtOH (b), EL (c) and GVL (d). The white squares correspond to the correlation functions where it was not possible to fit properly and the white circles to samples with a very low intensity of scattered light. The dotted lines should just indicate the evolution of the curves.

shown in Fig. 1. In every system two experimental lines were chosen $\left(B_{i}\right.$ and $\left.C_{i}\right)$. For a better comparison of the three solvents, the molar ratio of water-to-eco-solvent was kept constant. The solubilisation of DR-13 is expressed in units of optical density (O.D.). In Fig. 6 the O.D.s of the three solvents are plotted as a function of mass fraction. The addition of BA leads to an increase of solubility of DR-13 in the three systems. In Fig. 6(d) the dye solubility for the binary systems water-eco-solvent is plotted. The increase of O.D. is significant for EL and GVL with increasing eco-solvent concentration. A possible explanation for the different solubility properties could be the appearance or absence of clusters in the binary systems. EtOH forms no aggregates with water, so the solubility is very low. EL and GVL already form some cluster in water (see Fig. 4). This could be an argument for the higher solubility of DR-13.

But the eco-solvent concentration affects the DR-13 solubilisation as well. For samples with high amount of eco-solvent the increase of O.D. is not as remarkable as for lower concentrations.
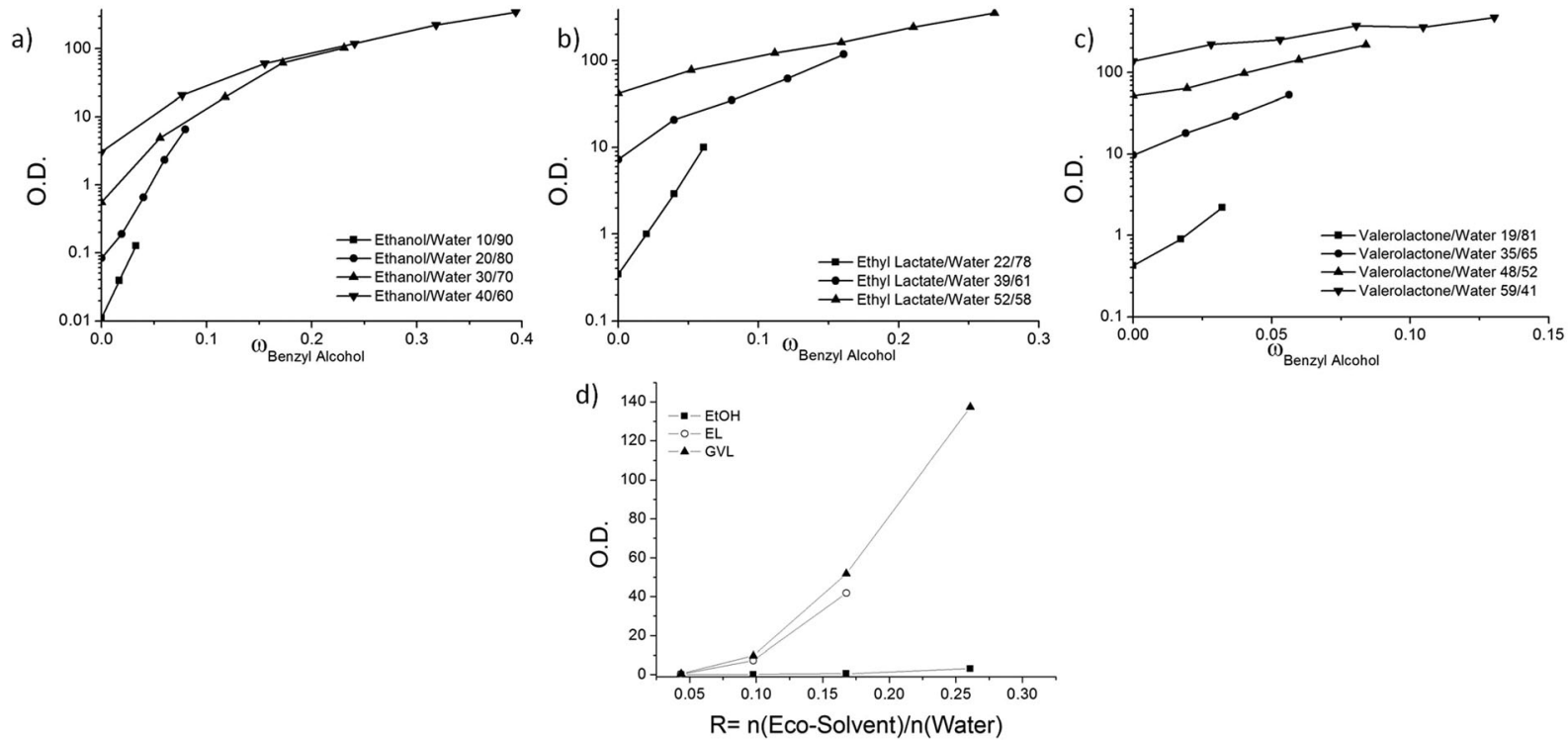

Fig. 6 DR-13 solubilisation as a function of the BA mass fraction for the systems water-ethanol (a), ethyl lactate (b), and $\gamma$-valerolactone (c). In the legend of every figure, the mass ratio of the eco-solvent-to-water is given corresponding to the dilution lines in Fig. 3. (d) shows the evolution of O.D. of the binary water-eco-solvent mixture. O.D. is plotted as a function of the molar ratio of eco-solvent-to-water. 

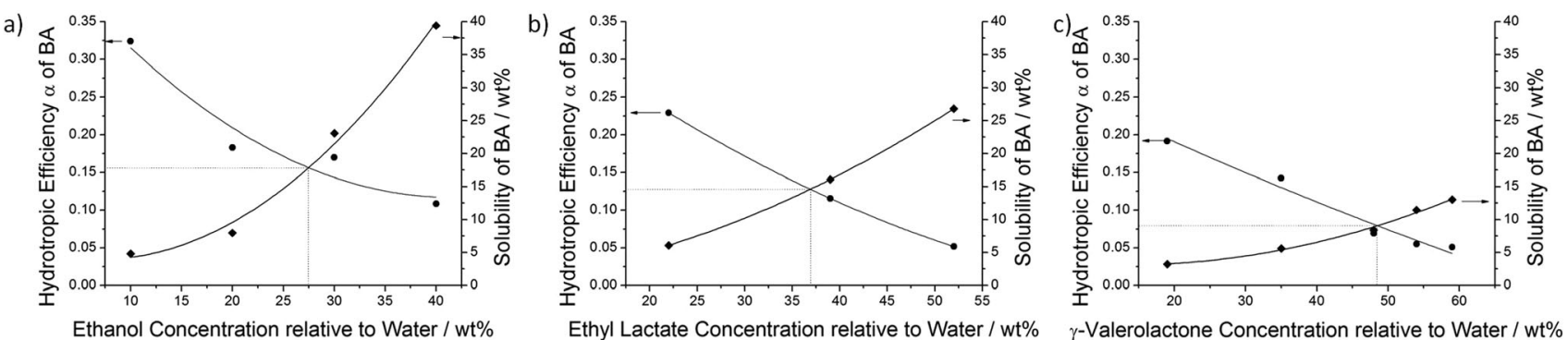

Fig. 7 Hydrotropic efficiency of BA in the systems water-EtOH (a), EL (b) and GVL (c) (circles) and BA solubility in the corresponding systems (diamonds) as a function of the eco-solvent to water ratio. The dotted lines show the crossing points of the two curves.

Bauduin et al. showed a way to characterize the hydrophobic efficiency $\alpha^{36}$ It is linked to the evaluation of the slopes of the linear part in the log-linear plots of the solubility of the hydrophobic dye, which is defined using the following equation:

$$
\alpha_{\mathrm{BA}}^{\mathrm{DR} 13}=\frac{\delta \log _{10} \text { O.D. }}{\delta[\mathrm{BA}]}
$$

In Fig. 7 a plot of the evolution of $\alpha$ for different water/ eco-solvent ratios is shown together with the solubility of BA in these systems. With increasing water/eco-solvent ratio the BA solubility increases as well but the hydrophobic efficiency decreases. Since this occurs for every eco-solvent this seems to be a general phenomenon which has already been described by Durand et al. ${ }^{25}$ Another important aspect of the obtained curves is the crossing point of the efficiency and solubility curve. It is shifted in the same manner. For a high hydrotropic efficiency low hydrotrope concentrations are necessary. In a recent study on the mechanism of hydrophobic drug solubilisation Booth et al. ${ }^{37}$ showed that self-association of hydrotropes decreases the solubilisation property. In other words, the selfassociation at high hydrotrope concentrations, which leads to a pronounced structure in the systems, as we showed with DLS and SLS measurements, leads to a decrease of hydrotropic efficiency. The results in Fig. 7 are experimental proof of this theory. Moreover, the absolute value of $\alpha$ in the water-eco-solvent binaries is highest for EtOH and decreases with EL and GVL. This is another hint that the self-aggregation of hydrotropes affects the facilitated hydrotropic efficiency.

\section{Conclusions}

In this paper we investigated the structuring and solubilisation properties of the ternary mixtures water-BA-eco-solvent. In these mixtures the solubility of the most hydrophobic molecule, in our case BA, was increased by the presence of the eco-solvent. In the single phase area, a region of optimal solubility located near the two-phase boundary was found. In this zone nano-structures occur. The sizes of these structures are too small to be considered as micelles. The appearance of micelles depends on the hydrophobicity of the solubilized molecule, and in the case of BA, this hydrophobicity is too small. ${ }^{19}$ Nevertheless, clusters of BA and eco-solvent are formed.
This was proved by the combination of DLS and SLS. In the presence of the eco-solvent the hydrotropic efficiency decreased with the increase of the degree of self-association of the ecosolvent in water. Comparing to the work of Booth et al., they only considered the simple direct hydrotropy, whereas in the presented paper the joint effects of two hydrotropes were used in order to investigate the concept of facilitated hydrotropy. This fact makes the two studies different. The present work can thus be considered as a generalisation and extension of Booth et al's work.

\section{References}

1 F. Chemat, M. A. Vian and G. Cravotto, Int. J. Mol. Sci., 2012, 13, 8615-8627.

2 H. Röper, Starch/Staerke, 2002, 54, 89-99.

3 C. Capello, U. Fischer and K. Hungerbühler, Green Chem., 2007, 9, 927-934.

4 L. Moity, M. Durand, A. Benazzouz, C. Pierlot, V. Molinier and J.-M. Aubry, Green Chem., 2012, 14(4), 1132-1145.

5 http://ethanolrfa.3cdn.net/d4ad995ffb7ae8fbfe_1vm62ypzd. pdf, 2012.

6 C. F. Runge and B. Senauer, How Biofuels Could Starve the Poor, May/June 2007.

7 C. S. M. Pereira, V. M. T. M. Silva and A. E. Rodrigues, Green Chem., 2011, 13, 2658-2671.

8 P. Delgado, M. T. Sanz, S. Beltrán and L. A. N. nez, Chem. Eng. J., 2010, 165(2), 693-700.

9 http://web.anl.gov/techtransfer/pdf/fact_sheets/ethyl_lac tate.pdf, 2006.

10 I. T. Horváth, Green Chem., 2008, 10, 1024-1028.

11 I. T. Horváth, H. Mehdi, V. Fabos, L. Boda and L. T. Mika, Green Chem., 2008, 10, 238-242.

12 L. Deng, J. Li, D. M. Lai, Y. Fu and Q. X. Guo, Angew. Chem., Int. Ed., 2009, 48, 6529-6532.

13 L. Deng, Y. Zhao, J. A. Li, Y. Fu, B. Liao and Q. X. Guo, ChemSusChem, 2010, 3, 1172-1175.

14 S. G. Wettstein, D. M. Alonso, Y. Chonga and J. A. Dumesic, Energy Environ. Sci., 2012, 5, 8199-8203.

15 Z. P. Yan, L. Lin and S. J. Liu, Energy Fuels, 2009, 23, 3853-3858.

16 X. L. Du, L. He, S. Zhao, Y. M. Liu, Y. Cao, H. Y. He and K. N. Fan, Angew. Chem., Int. Ed., 2011, 50, 7815-7819. 
17 T. Werpy and G. Petersen, National Renewable Energy Laboratory, Golden, CO Technical Report, Report No. NREL/TP-510-35523, 2004.

18 J. J. Bozell and G. R. Petersen, Green Chem., 2010, 12, 539-554.

19 M. L. Klossek, D. Touraud, T. Zemb and W. Kunz, ChemPhysChem, 2012, 13(18), 4116-4119.

20 G. D. Smith, C. E. Donelan and R. E. Barden, J. Colloid Interface Sci., 1977, 60(3), 488-496.

21 N. F. Borys, S. L. Holt and R. E. Barden, J. Colloid Interface Sci., 1979, 71(3), 526-532.

22 B. A. Keiser, D. Varie, R. E. Barden and S. L. Holt, J. Phys. Chem., 1979, 83(10), 1276-1280.

23 B. A. Keiser and S. L. Holt, Inorg. Chem., 1982, 21, 2323-2327. 24 R. Schott and A. Pfennig, Mol. Phys., 2004, 102(4), 331-339.

25 M. Durand, A. Stoppa, V. Molininer, D. Touraud and J.-M. Aubry, J. Solution Chem., 2012, 41, 555-565.

26 B. Gupta, D. S. Mishra, C. H. Cheng and S. H. Yalkowsky, Toxicol. Environ. Chem., 1991, 33, 7-21.

27 Solubility and Solubilization in Aqueous Media, ed. S. H. Yalkowsky, American Chemical Society Publication, 1 edn, 1999.
28 K. L. Bhat, A. Garg and C. W. Bock, Dyes Pigm., 2002, 52, 145-159.

29 P. Simamora, J. M. Alvarez and S. H. Yalkowsky, Int. J. Pharm., 2001, 213, 25-29.

30 M. Clausse, L. Nicolas-Morgantini, A. Zradba and D. Touraud, Surfactant Science Series, Microemulsion Systems, Dekker, New York, 1987.

31 E. Moreels, W. D. Ceuninck and R. Finsy, J. Chem. Phys., 1987, 86(2), 618-623.

32 H. Wahid, J. Mol. Liq., 1993, 55, 1-18.

33 B. Chu, Laser Light Scattering, Academic Press, London, 2nd edn, 1991.

34 M. Corti and V. Degiorgio, J. Phys. Chem., 1981, 85, 711-717.

35 T. Zemb, M. Drifford, M. Hayoun and A. Jehanno, J. Phys. Chem., 1983, 87(22), 4524-4528.

36 P. Bauduin, A. Renoncourt, A. Kopf, D. Touraud and W. Kunz, Langmuir, 2005, 21, 6769-6775.

37 J. J. Booth, S. Abbott and S. Shimizu, J. Phys. Chem. B, 2012, 116(51), 14915-14921. 